\title{
Doppler Imaging as a Test for Binary Star Formation Theories
}

\author{
Klaus G. Strassmeier \\ Institute for Astronomy, University of Vienna, A-1180 Vienna, Austria
}

\begin{abstract}
Photospheric Doppler images for both stellar components of the double-lined spectroscopic binary V824 Ara reveal surface temperature inhomogeneities of up to $1800 \mathrm{~K}$ on both stars. The absolute brightness and the mass of the two stars suggest that they are very close to the main sequence - but not yet on the ZAMS - if an inclination of the orbital plane (and rotational axes) of $52^{\circ}$ is adopted as suggested by the Doppler imaging. Since both stars are active, we solve for the inclinations of the rotation axes of both stellar components separately and emphasize that it can be used to test coplanarity predictions from binary star-formation theories. We find that the spin inclinations for the individual components of V824 Ara agree to within their uncertainties.
\end{abstract}

\section{Introduction}

Doppler imaging of rapidly-rotating stars is a powerful technique to resolve their surface temperature inhomogeneities, also known as starspots. Periodic variations in the spectral line profiles during a star's rotation are numerically inverted into a spherical stellar surface temperature with the help of tomographic algorithms. The technique is nowadays applied to a large variety of rotating stars ranging from single G-type bright giants to binary $M$ dwarfs in close binaries. A recent summary of Doppler images was given in Strassmeier (2000) and is available on the web (www.astro.univie.ac.at/ $\sim \mathrm{kgs} / \mathrm{DI} / \mathrm{summary} /$ ). Out of the 47 stars in this sample 20 were in close binaries, almost all of the RS CVn or BY-Dra type. Seven weak-lined T Tauri's (WTTS) and three classical T Tauri's are also included. However, only one WTTS binary has been mapped so far: the double-lined G5 (IV) + K0 (IV-V) star V824 Ara = HD 155555 (Hatzes \& Kürster 1999, Strassmeier \& Rice 2000).

\section{The Idea}

If we are able to separate the spectra of both components of a double-lined young binary system, we may apply the Doppler imaging technique to both stars separately and determine their respective inclinations of the rotational axis independently from each other. This, in turn, may allow the detection of a deviation from coplanarity, a quantity that is otherwise not observable. So far, V824 Ara is the only double-lined young binary that fulfills the stringent 

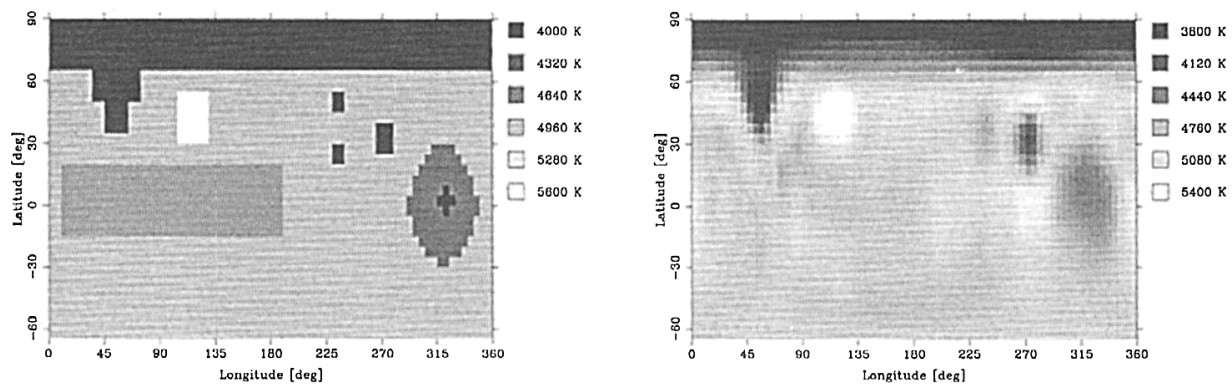

Figure 1. A comparison of an artificial input map (left) and the reconstruction. The $\mathrm{S} / \mathrm{N}$ was adopted to be 3000:1 in order to separate the inclination effect from other internal errors like uncertain damping constants, microturbulence effects, continuum-setting errors etc.. Even in the presence of a large number of possible errors, the maps can be reconstructed amazingly reliable.

requirements for Doppler imaging (spottedness, rapid rotation, relatively bright, medium inclination, no binary interactions).

Before a comparison with theory is made I emphasize to be aware of the following shortcomings of this idea:

- The age of a field star/binary is notoriously uncertain.

- The inclination angle from Doppler imaging is only good to within, say, $\pm 10^{\circ}$.

In the following section we will explore these two concerns in more detail before we go on to map the components of V824 Ara.

\section{Testing the Inclination Sensitivity of Doppler Imaging}

In this test, we adopt an artificial star with an arbitrary inclination but use a different inclination in the reconstruction of its surface spot distribution. We first solve the forward problem, i.e. create the "data" from the input star with known parameters, add some random noise and specific external errors like straylight, continuum offsets etc. (see Rice \& Strassmeier 2000), and then invert the "data" to create a Doppler image. The latter can then be compared with the original input map (Fig. 1). We run our line-profile inversion code TEMPMAP (see Rice 1996) on artificial data with high $\mathrm{S} / \mathrm{N}$ ratio and in steps of $5^{\circ}$ from $i=5^{\circ}$ to $i=85^{\circ}$. Fig. 2 demonstrates that the correct inclination $\left(i=60^{\circ}\right)$ can be recovered just from the change of the $\chi^{2}$ as a function of $i$. The width of the minimum in Fig. 2 allows the reconstruction of inclination values of $\pm 2.5^{\circ}$ of the nominal value for a width of 1- $\sigma$ of the minimum $\chi^{2}$ and thus verifies the principle usefullness of this method.

Hatzes et al. (1996) had noticed that low-inclination active stars had a more pronounced flat-bottomed line-profile shape compared to high-inclination stars. This is understandable when a star has spots at or near the rotational pole 
because the Doppler span over which flux is missig due to the cool spots gets wider the lower the inclination. Our simulations fully confirm Hatzes' conclusions. The line-profile shape is thus an indicator for the inclination of the stellar rotation axis and the quality of our fits to real data with limited $\mathrm{S} / \mathrm{N}$ ratio and with numerous external errors, e.g. in the flat field, is such that we can achieve an inclination to within $\pm 10-15^{\circ}$.

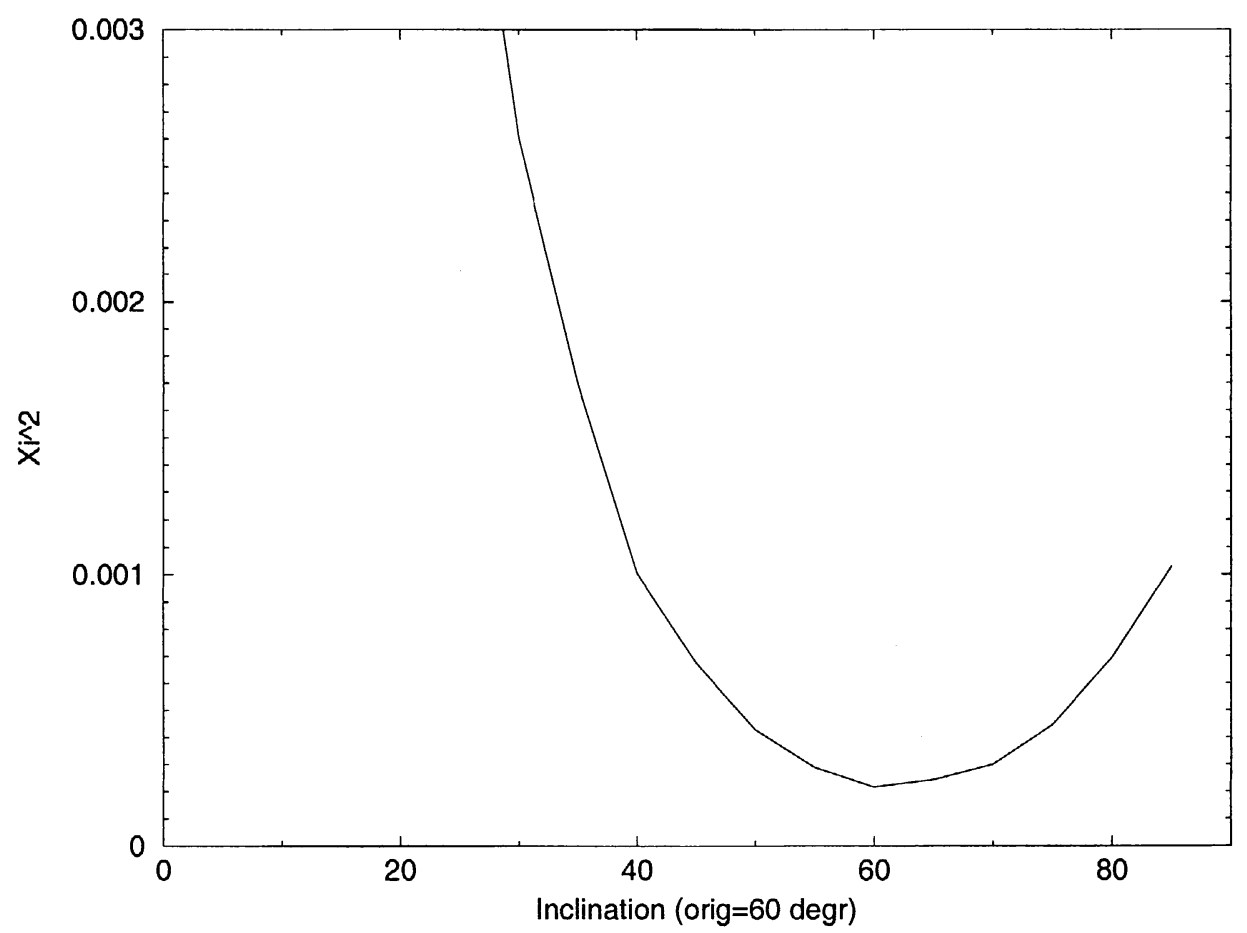

Figure 2. Reconstruction of the artificial spot distribution in Fig. 1 with inclination angles between $5^{\circ}$ and $85^{\circ}$. The vertical axis is $\chi^{2}$. A clear minimum near the correct inclination is obtained.

From its high lithium abundance of nearly the primordial value, Pasquini et al. (1991) suggested that the binary is part of the young disk population. There is also a nearby M-star companion (LDS 587B) $33^{\prime \prime}$ away. With this visual companion showing also very high levels of activity and also high lithium abundance (Martin \& Brandner 1995) it seems likely that the V824 Ara + LDS587B system is indeed pre-main sequence.

Further constraints come from the parallax measurement by the Hipparcos satellite that revised the distance of V824 Ara to $31.4 \pm 0.8 \mathrm{pc}$ and thus $M_{V}=$ $+4.34 \pm 0.05$ mag. Together with the components' magnitude difference of 0.97 measured from the equivalent-width ratio in the CORAVEL bandpass (Pasquini et al. 1991), one finds the individual components' absolute magnitudes to be $M_{V}(G 5)=+4^{\mathrm{m}} 73$ and $M_{V}(K 0)=+5.60$. The propagated error from the parallax and the brightness difference results in an uncertainty of just $\pm 00^{\mathrm{m}} 05$ for both components, but assumes no error for the apparent brightness adopted. 
Both components' relatively high luminosities of 1.07 and $0.54 L_{\odot}$ (obtained with the bolometric corrections from Flower 1996), together with their high lithium abundances, then suggests them to be in the process of arriving on the ZAMS rather than leaving it.

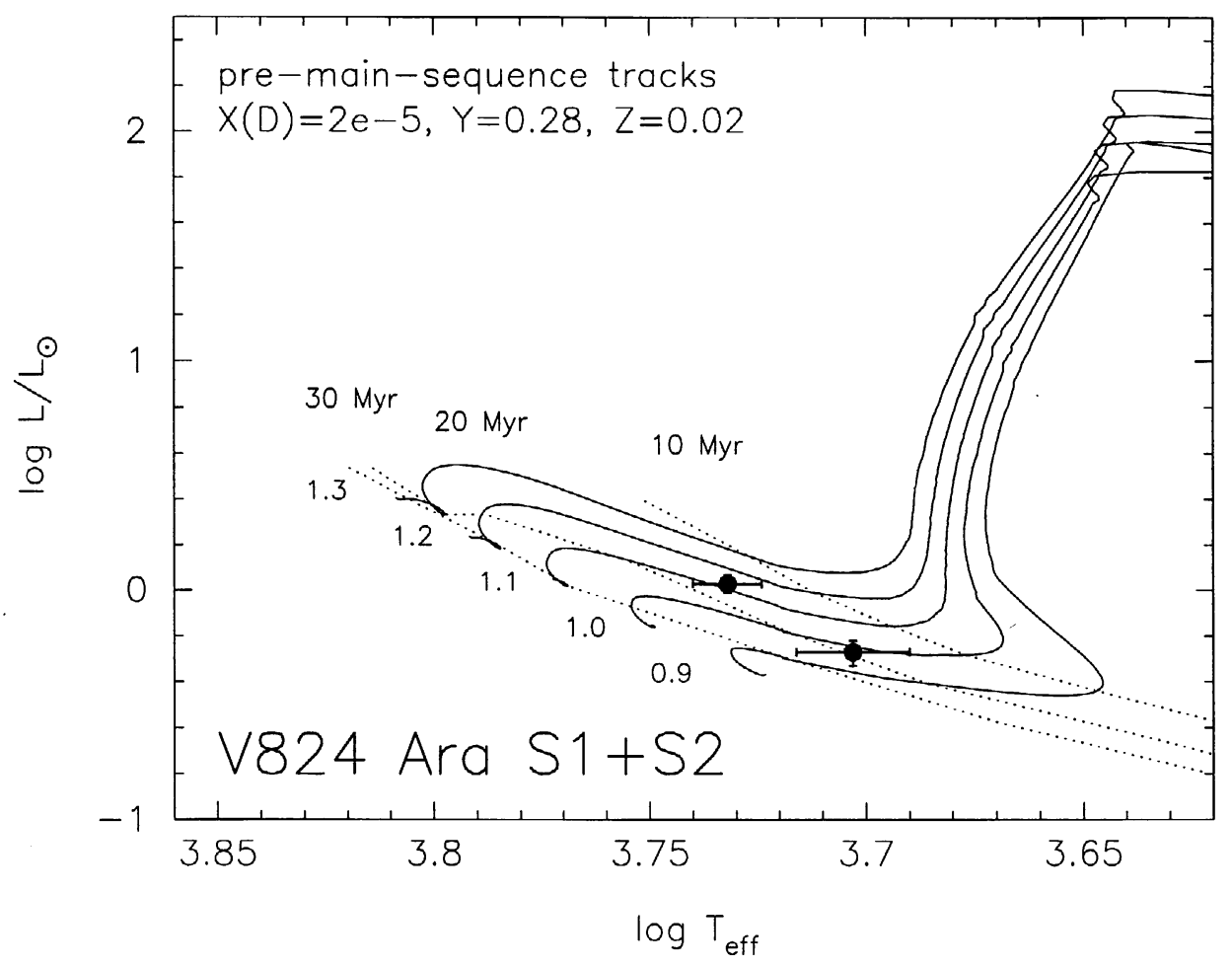

Figure 3. The position of V824 Ara S1+S2 in the H-R diagram. The solid lines are the pre-main-sequence tracks of D'Antona \& Mazzitelli (1997) for masses of $0.9,1.0,1.1,1.2$, and 1.3 solar masses. The dotted lines are isochrones for 10,20 , and $30 \mathrm{Myr}$. The suggested age of V824 Ara is thus approximately $18 \mathrm{Myr}$.

Fig. 3 shows the position of the two stellar components in the H-R diagram with respect to the pre-main-sequence tracks from D'Antona \& Mazzitelli (1997). The formal comparison in Fig. 3 gives masses of $1.12 \mathrm{M}_{\odot}$ and $0.99 \mathrm{M}_{\odot}$ for the primary and secondary, respectively. However, as warned several times by D'Antona \& Mazzitelli, such a straightforward comparison may not be conclusive because of the remaining theoretical uncertainties from the Deuterium-burning process and the convection treatment. At the moment though, the low error level for the V824 Ara observations - due to the precise orbit and the Hipparcos parallax - are not in agreement with the star being already off the ZAMS. The isochrone that fits both stars best suggests an age of $\approx 18$ Myr for V824 Ara (Fig. 3). 
V824 Ara Primary
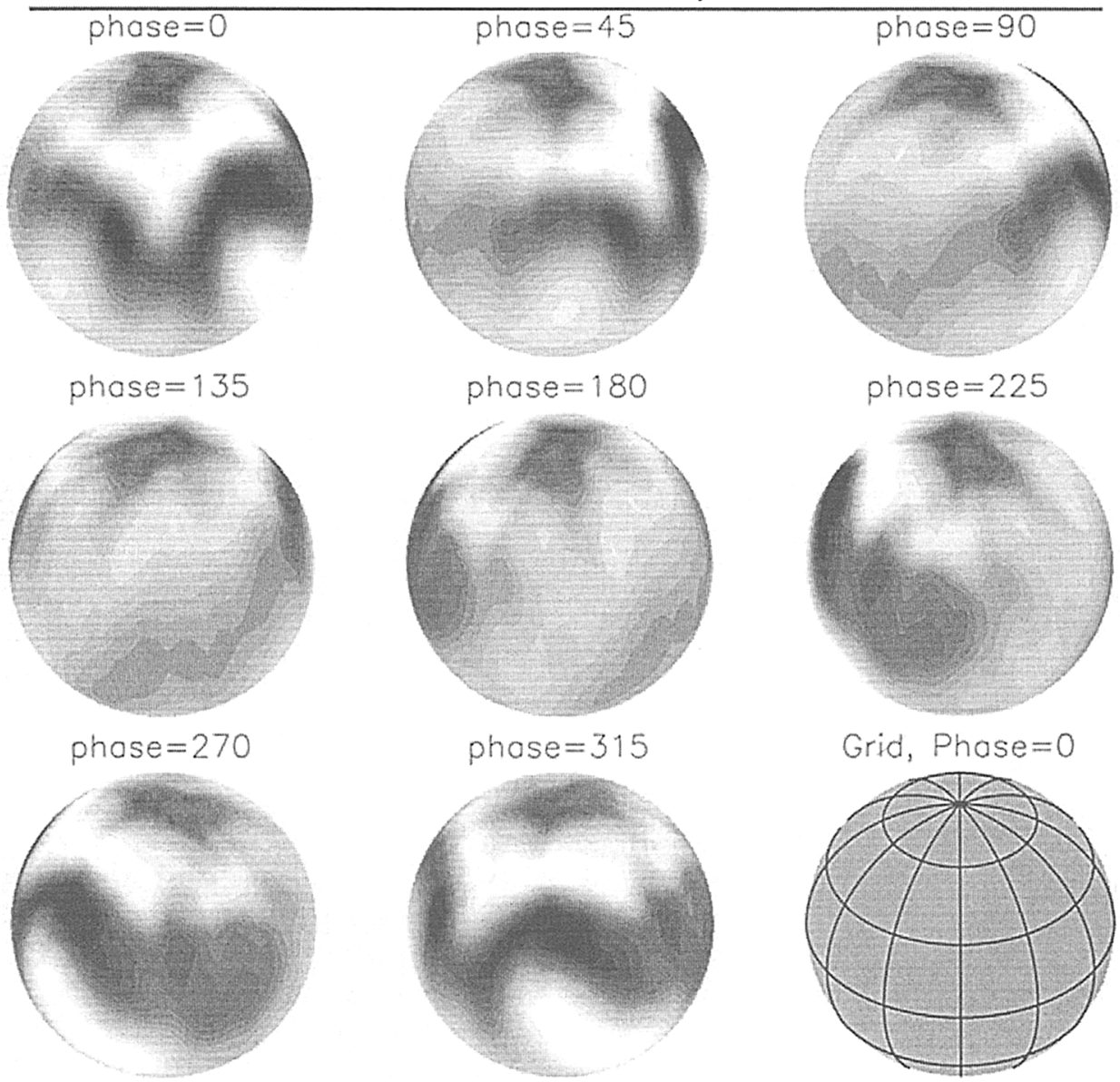

$3370 K$

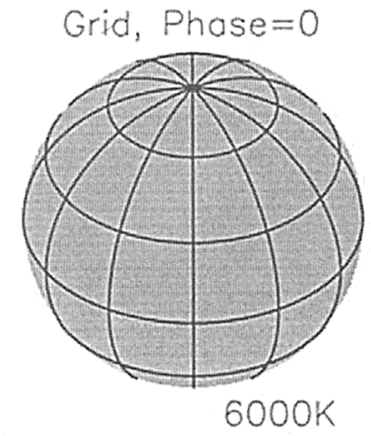

Figure 4. Doppler image of the primary of V824 Ara in May 1996. A spherical projection style at eight phases in steps of $45^{\circ}\left(0^{\mathrm{p}} 125\right)$ is adopted. A dummy in the lower right corners shows the surface grid.

\section{The Case of V824 Ara}

\subsection{Individual Doppler Maps}

Fig. 4 and 5 show our Doppler images of both components of V824 Ara. These maps were derived with an inclination of $52^{\circ}$ and $v \sin i$ of $36.8 \pm 1 \mathrm{~km} \mathrm{~s}^{-1}$ and $33.7 \pm 1.5 \mathrm{~km} \mathrm{~s}^{-1}$ for the primary and secondary component, respectively, as well as micro- and macroturbulences of $2.0 \mathrm{~km} \mathrm{~s}^{-1}$ with solar abundances.

The range of surface temperatures on the primary is $3400-6000 \mathrm{~K}$ with an average (surface-integrated) value of $4900 \mathrm{~K}$ while the secondary shows a range 

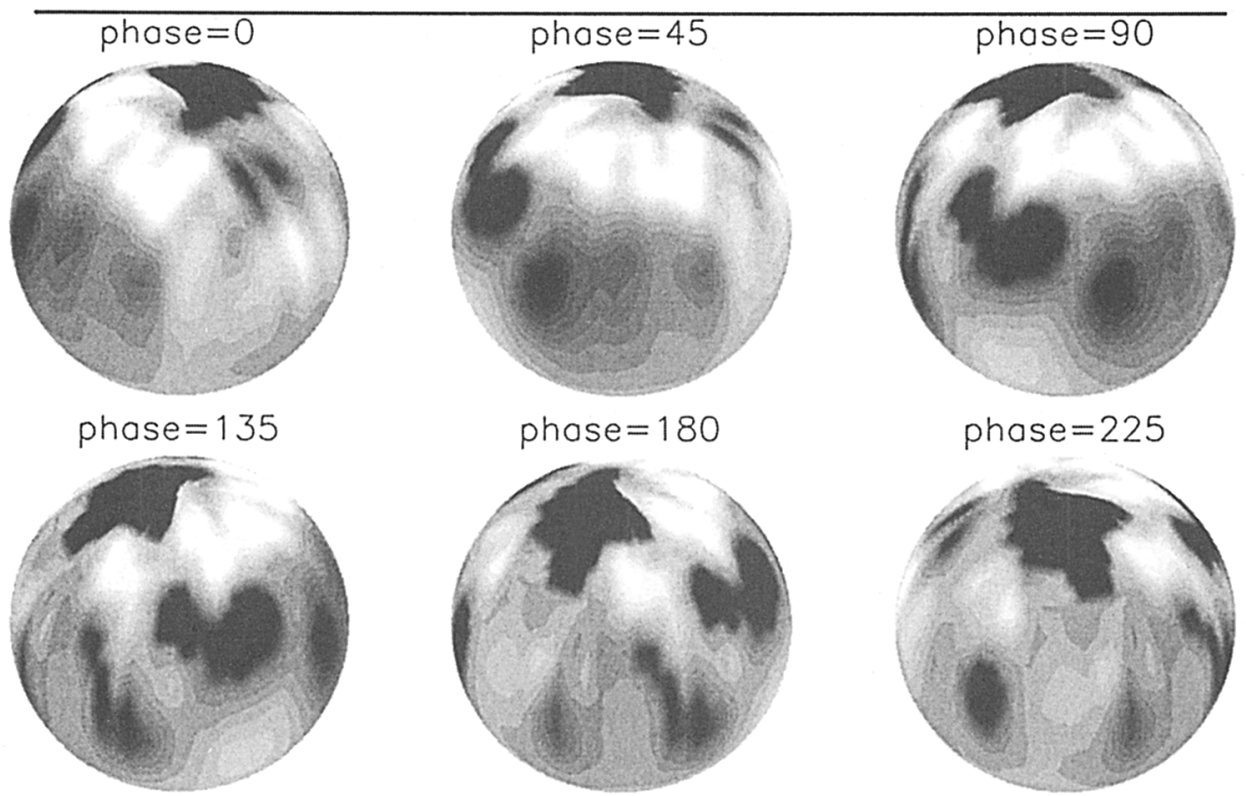

phase $=225$

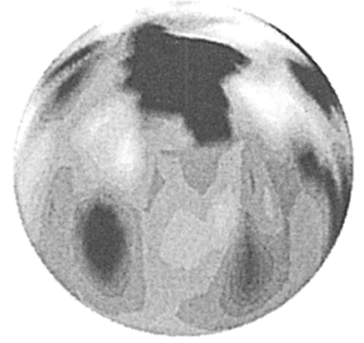

phase $=270$

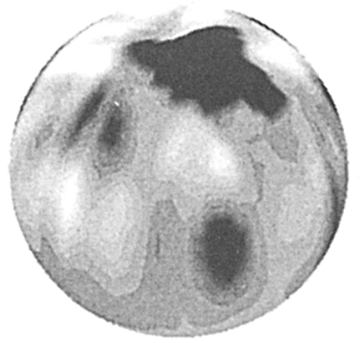

phase $=315$

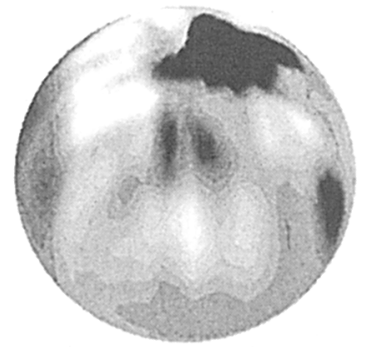

$3370 \mathrm{~K}$

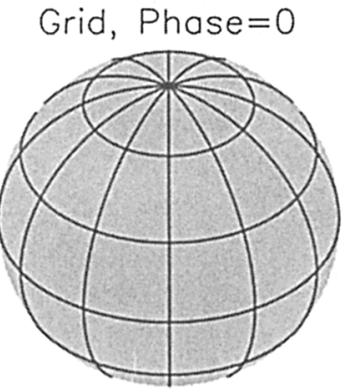

$6000 \mathrm{~K}$

Figure 5. Doppler image of the secondary of V824 Ara. Otherwise as in Fig. 4.

of 3300-6000 $\mathrm{K}$ and an average of $4550 \mathrm{~K}$. The most significant feature on the primary is a complex equatorial region covering the longitudes between $\ell \approx 270^{\circ}$ and about $\ell \approx 360^{\circ}$. Our primary-star maps also recover a cool and slightly asymmetric polar spot with a temperature difference of approximately $1700 \mathrm{~K}$ relative to the adopted "unspotted" photosphere of $5400 \mathrm{~K}$. The secondary star does not show a full polar cap-like spot but instead has a cool, very high-latitude spot.

The spots seem to group near $270^{\circ}$ on the primary and $90^{\circ}$ on the secondary, i.e. exactly the opposite distribution than that of the two polar spots seen by Hatzes \& Kürster (1999) in 1990. However, we fully confirm the finding by Hatzes \& Kürster (1999) that the low-latitude spots on both V824 Ara com- 


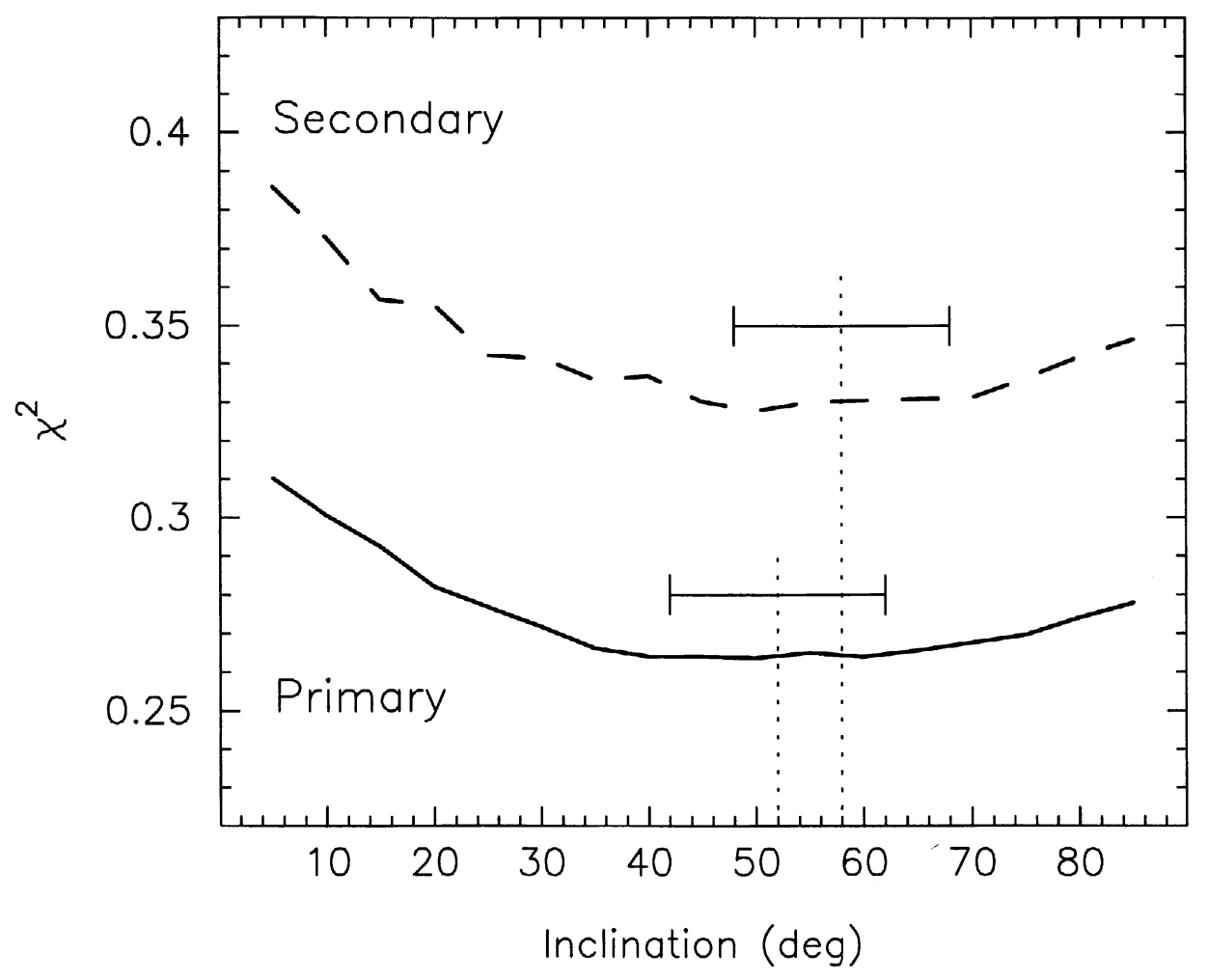

Figure 6. The misfit between the data and the predicted profiles $\left(\chi^{2}\right)$ as a function of stellar inclination. The full line is for the primary, the dashed line for the secondary. The most likely values are indicated with a vertical dotted line, and the range of possible values is shown as horizontal bars. Note that the ranges significantly overlap, which suggests that the inclinations are very likely identical for both components.

ponents are preferentially located on the anti-facing hemispheres. This suggests that the spots on V824 Ara tend to be located preferentially along the apsidal line.

\subsection{The Spin Inclinations of the Binary Components}

We now repeat the analysis in Sect. 3.1 but instead of artificial data we use our data for V824 Ara. Images are computed in steps of $5^{\circ}$ from $5^{\circ}$ to $85^{\circ}$ and the results are shown in Fig. 6 . The figure shows the $\chi^{2}$ distribution from the fits from the Doppler images of both components. Both distributions show a flat minimum of width $\approx 20^{\circ}$ at about the same inclination range, $42^{\circ}-62^{\circ}$ for the primary and $48^{\circ}-68^{\circ}$ for the secondary component. The formal values are found from a low-order polynomial fit to the $\chi^{2}$ curves and are $52^{\circ}$ for the primary and $58^{\circ}$ for the secondary. The quality and sampling of the present data limit the precision to which the inclination can be determined. We do not regard the formal difference of $6^{\circ}$ as significant. Therefore, we conclude that the rotation 
axes of the two components are aligned and perpendicular with respect to the orbital plane and adopt $52^{\circ}$ as the most likely inclination.

\section{Discussion and Summary}

For main-sequence and post-main-sequence binaries, it has been already argued that the asynchronuously rotating active components of the RS CVn- and BY Dra binaries may be a direct cause of a misalignment of their rotational axes (e.g. Glebocki \& Stawikowski 1997). Imprecise $v \sin i$ measurements and very uncertain stellar radii for giants and subgiants, however, still prevent a verification of this suggestion.

We have shown that - under favorable circumstances - the Doppler imaging technique can be used to determine precise inclination angles for individual stars in a non-eclipsing close binary. Our application to the components of the 18Myr young close binary V824 Ara showed that their rotation axis are most likely coaligned and perpendicular to the orbital plane.

Acknowledgments. I am grateful to the Austrian Science Foundation for their support with grants S7301 and S7302 and, of course, to the Potsdam staff and especially Hans for organizing such a pleasant meeting. It is always a pleasure to acknowledge the collaboration with J. B. Rice from Brandon University.

\section{References}

D'Antona, F., \& Mazzitelli, I. 1997, in Cool stars in clusters and associations, ed. R. Pallavicini \& G. Micela, Mem. S. A. It., 68, 807

Flower P. J. 1996, ApJ 469, 355

Glebocki, R., \& Stawikowski, A. 1997, A\&A, 328, 579

Hatzes, A. P. 1995, in IAU Symp. 176, Poster Proceedings: Stellar Surface Structure, ed. K. G. Strassmeier, Univ. Vienna, 90

Hatzes, A. P., \& Kürster, M. 1999, A\&A, 346, 432

Hatzes, A. P., Vogt, S. S., Ramseyer, T. F., \& Misch, A. 1996, ApJ, 469, 808

Martin, E. L., \& Brandner, W. 1995, A\&A, 294, 744

Pasquini, L., Cutispoto, G., Gratton, R., \& Mayor, M. 1991, A\&A, 248, 72

Piskunov, N. E., Vincent, A., Duemmler, R., Ilyin, I., \& Tuominen, I. 2000, A\&A, submitted

Rice, J. B. 1996, in IAU Symp. 176, Stellar Surface Structure, ed. K. G. Strassmeier \& J. L. Linsky, Kluwer Acad. Press, 19

Rice, J. B., \& Strassmeier, K. G. 2000, A\&AS, submitted

Strassmeier, K. G., \& Rice, J. B. 2000, A\&A, in press

Unruh, Y. C., Collier Cameron, A., \& Guenther, E. 1998, MNRAS, 295, 781 